\title{
Assurance maladie en Suisse : les assurances supplémentaires nuisent-elles à la concurrence sur l'assurance de base?
}

\author{
Brigitte Dormont *, Pierre-Yves Geoffard ** et Karine Lamiraud ***
}

De nombreux pays ont introduit des mécanismes concurrentiels en assurance maladie, tout en instaurant une régulation pour éviter la sélection des risques et garantir une solidarité entre malades et bien portants. Le modèle de la " concurrence régulée » est ainsi appliqué pour l'assurance maladie de base en Suisse, aux Pays-Bas, en Allemagne, en Israël. Il inspire la réforme de l'assurance maladie aux États-Unis. Cet article analyse le fonctionnement d'un tel système en considérant le cas de la Suisse, où l'on s'intéresse aux interférences potentielles entre le marché des assurances supplémentaires et celui de l'assurance de base. L'organisation actuelle de l'assurance maladie en France diffère de celle du système suisse. Mais la question d'une régulation du marché des assurances complémentaires pourrait être posée à terme.

Douze ans après l'introduction de la concurrence régulée, les résultats observés en Suisse sont décevants. Bien que les écarts de primes constatés entre les compagnies d'assurance soient très importants, la proportion d'assurés qui changent de caisse reste faible. Tout se passe comme si les assurés ne faisaient pas jouer la concurrence.

Notre analyse montre que la faible mobilité des assurés résulte de la coexistence de deux marchés d'assurance maladie soumis à des règles différentes : le marché de l'assurance de base, où la sélection des risques est interdite, et celui de l'assurance supplémentaire, où elle est autorisée. Les estimations montrent que la propension à changer de caisse est beaucoup plus faible chez les détenteurs d'une assurance supplémentaire qui estiment que leur santé n'est pas excellente. Comme il est préférable pour des raisons pratiques d'avoir son assurance de base et son assurance supplémentaire dans la même caisse, il existe un lien de fait entre les deux marchés. Le droit de sélectionner les candidats à la souscription pour l'assurance supplémentaire nuit à la concurrence sur l'assurance de base.

\footnotetext{
${ }^{*} P S L$, Université Paris-Dauphine (LEDa-LEGOS) et Cepremap

${ }^{* *}$ Paris School of Economics (CNRS), EHESS

***ESSEC Business School, THEMA-Université de Cergy Pontoise

Ce travail a bénéficié d'un soutien du Fonds National suisse et de la Chaire Santé, placée sous l'égide de la Fondation Du Risque (FDR) en partenariat avec PSL, Université Paris-Dauphine, I'ENSAE et la MGEN. Les auteurs tiennent à remercier la Swiss School of Public Health+ qui a financé l'administration du questionnaire qu'ils ont élaboré.
} 
$\mathbf{L}$ a fédération helvétique comporte 7,7 millions d'habitants répartis dans 26 cantons. L'offre de soins et la politique de santé publique sont définies au niveau des cantons mais l'assurance maladie est organisée au niveau fédéral. Elle comporte deux niveaux : une assurance de base obligatoire et des assurances supplémentaires facultatives. L'assurance de base peut être souscrite auprès de multiples compagnies : le système suisse privilégie la concurrence entre caisses d'assurance en favorisant le libre choix du consommateur.

Une assurance maladie obligatoire est en principe définie pour un périmètre de soins donné, le «panier de base». On désigne ainsi des soins jugés fondamentaux, auxquels tous les membres de la société doivent avoir accès sans restriction. Le contenu du panier de base n'est pas figé : il évolue en fonction des normes sociales et des possibilités offertes par les progrès médicaux. Par exemple, pour la France et nombre de pays européens, les soins exclus du panier de base correspondent à la médecine alternative, au confort à l'hôpital et aux dépassements d'honoraires. Dans les systèmes de soins de la plupart des pays développés, l'assurance de base obligatoire coexiste avec des assurances facultatives, qui jouent le rôle d'assurances complémentaires lorsqu'elles participent à la couverture des soins de base et celui d'assurances supplémentaires lorsqu'elles couvrent les autres soins. En France, ce qu'on appelle les organismes d'assurances complémentaires cumulent les fonctions d'assurances complémentaire et supplémentaire. Leur fonction principale est celle d'assurance complémentaire, c'est-à-dire de coassurance des soins de base, ce qui constitue une originalité française. En effet, en Suisse, et dans les pays de l'OCDE dotés d'une assurance de base obligatoire, les assurances facultatives assument plutôt la fonction d'assurance supplémentaire (Colombo et Tapay, 2004).

L'objet de cet article est d'analyser le fonctionnement d'un système de concurrence régulée en assurance maladie. En considérant le cas de la Suisse, on s'intéresse aux interférences potentielles entre le marché des assurances supplémentaires et la concurrence sur le marché de l'assurance de base. L'organisation actuelle de l'assurance maladie en France diffère de celle du système suisse. Mais les assurances complémentaires participent de plus en plus à la couverture des soins dans notre pays. La question de la régulation du marché des assurances complémentaires doit donc être posée.
L'analyse du système suisse, qui s'appuie sur un marché régulé d'assurance maladie, peut illustrer certains enjeux du système français.

\section{La concurrence régulée en assurance maladie : l'exemple de la Suisse}

En Suisse, pour l'année 2007, l'assurance de base et les assurances supplémentaires ont couvert respectivement $35,3 \%$ et $9,2 \%$ des dépenses de santé. Une part non négligeable des coûts - 30,7\% - est à la charge directe des ménages ${ }^{1}$. Le reste du financement correspond aux budgets cantonaux consacrés aux établissements de santé (OFS, 2009). En France pour la même année 2007, la Sécurité sociale de base a couvert 76,2\% des dépenses de santé et les organismes complémentaires 13,5\%. Dans notre pays, les ménages paient directement 8,9\% de leurs dépenses de santé (DREES, 2010).

L'offre d'assurance maladie par une pluralité de compagnies est une caractéristique du système suisse depuis près d'un siècle. La réforme fédérale introduite en 1996 par la loi sur l'Assurance Maladie(LAMal) rend obligatoire l'assurance de base pour les résidents suisses. Elle organise pour le marché de cette assurance une concurrence qui était jusqu'alors très peu régulée.

Le principe de cette réforme est de faciliter les changements de caisse pour les affiliés afin d'inciter les assureurs à fournir le meilleur service au moindre coût. Le dispositif mis en place favorise les mécanismes concurrentiels :

- l'assurance de base est obligatoire ;

- tout assureur doit offrir un contrat à un individu qui en fait la demande ;

- les contrats sont standardisés : le contenu du panier de base, les niveaux des franchises et les taux de co-paiement sont définis de façon homogène pour tous les assureurs ;

- les primes ne peuvent pas être ajustées en fonction des dépenses de soins de l'assuré : elles doivent être uniformes pour tous les individus de la même zone géographique ayant opté

\footnotetext{
1. L'importance des sommes à la charge des ménages en Suisse est liée à l'existence de franchises et de co-paiements. En outre, aucun soin d'optique ou de dentaire n'est inclus dans le panier de soins de l'assurance de base.
} 
pour le même niveau de franchise et le même type de contrat ${ }^{2}$;

- l'assurance ne peut pas être financée par l'employeur, la prime est payée intégralement par le souscripteur.

L'obligation d'assurance permet d'instaurer un partage du risque maladie à l'échelle de la population, réduisant le coût de l'assurance pour chacun. La standardisation des contrats a pour intérêt de définir une concurrence en prix seulement: seul le niveau des primes différencie une compagnie d'une autre, facilitant les arbitrages du consommateur. L'information sur les primes est largement diffusée dans la presse et sur internet. Le fait que la prime d'assurance maladie soit intégralement payée par le souscripteur est prévu pour rendre les individus plus sensibles au coût et donc plus réactifs aux différences de primes entre les compagnies.

Imposer une prime uniforme, indépendante de l'état de santé des individus, permet à la solidarité entre malades et bien portants de jouer. Selon des principes d'équité verticale, la loi prévoit aussi que les primes d'assurance des individus les plus pauvres soient partiellement prises en charge par chaque canton sous forme de subsides. Cette disposition vise à rendre effective l'obligation de s'assurer.

La LAMal cherche à bannir les stratégies de sélection des risques. Tout d'abord, elle oblige chaque assureur à satisfaire toute demande de contrat. Ensuite, un mécanisme de compensation des risques est instauré, qui décourage la chasse aux bons risques en permettant aux caisses couvrant une population plus âgée de recevoir un financement de la part de caisses dont les affiliés présentent une structure démographique plus favorable ${ }^{3}$. L'imposition d'un contrat standard joue aussi son rôle : elle évite la sélection des risques qui passerait par une stratégie de ciblage de publics particuliers à travers la segmentation des contrats (contrats jeunes, low-cost, etc., stratégie présente dans le secteur des complémentaires en France).

Pour offrir un niveau de prime compétitif, les compagnies doivent réduire leurs coûts de gestion. En revanche, il leur est difficile de constituer un réseau de professionnels médicaux attitrés avec une contractualisation formalisant un engagement à délivrer des soins de façon efficiente. En effet, les caisses ont l'obligation de contracter avec tous les prestataires de soins : en dehors des contrats de type «HMO ${ }^{4} »$, peu développés en Suisse, les assurés peuvent consulter le médecin de leur choix.

Obligation d'assurance, interdiction de la sélection, fixation de primes indépendantes des caractéristiques de la personne, standardisation des contrats et compensation des risques : ces principes de régulation de la concurrence en assurance maladie sont appliqués, avec des variantes nationales, en Suisse, aux Pays-Bas, en Allemagne et en Israël. Ils inspirent la récente réforme de l'assurance maladie aux États-Unis (Affordable Care Act, mars 2010).

Nous l'avons vu, cette régulation concerne un périmètre de soins donné, le panier de base. Dès lors, il est possible qu'émerge une offre d'assurance pour couvrir les autres soins. En Suisse, les assurés ont la possibilité de souscrire des assurances supplémentaires comme par exemple l'assurance soins dentaires, médecines alternatives, hospitalisation en chambre simple ou double. Un assuré peut souscrire son assurance obligatoire et ses éventuels contrats supplémentaires auprès de la même caisse ou auprès de caisses différentes.

Le législateur a prévu une séparation stricte de l'assurance de base et de l'assurance supplémentaire. Elles sont régulées par des lois différentes, l'assurance de base étant réglementée par la LAMal et les assurances supplémentaires par la Loi sur les Contrats d'Assurance (LCA). Différence importante, la sélection des risques est autorisée pour les assurances supplémentaires. En effet, un questionnaire de santé peut être présenté pour toute demande de souscription.

Depuis la mise en place de la LAMal, le nombre de compagnies reconnues au niveau fédéral pour l'assurance de base a diminué, de 159 en 1996 à 87 en 2007. Mais le nombre d'assureurs par canton a augmenté. À deux exceptions près (les Grisons et le Valais), le nombre de caisses

2. Il existe des contrats avec des restrictions sur le choix du médecin. Il y a aussi trois tranches d'âge permettant de distinguer les enfants et les jeunes : 0-18 ans, 19-26 ans, plus de 26 ans.

3. Le mécanisme de compensation des risques actuellement en vigueur est fondé sur une prévision de dépenses individuelles de santé basée sur l'âge et le sexe de l'assuré. Un troisième facteur d'ajustement, le risque de maladie grave (évalué par un séjour hospitalier de plus de trois jours) a été adopté par le parlement le 21 décembre 2007 et est entré en vigueur le 1er janvier 2012.

4. Les contrats "HMO » se réfèrent au modèle américain de Health Maintenance Organization : il s'agit d'organisations où l'assurance maladie et la fourniture de soins sont intégrées. Ces organisations mettent en place une contractualisation qui vise à intéresser les médecins aux résultats financiers de la compagnie. Ils ont alors un intérêt direct à fonctionner de façon efficiente. 
opérant par canton était compris entre 36 et 40 en 1996. En 2007, le nombre de caisses par canton varie de 50 à 64 caisses.

Les dispositifs favorisant la mobilité des assurés et le nombre élevé de compagnies devaient créer une forte concurrence au sein de chaque canton et conduire à un alignement des primes au niveau le plus bas. Encore faut-il que la pression concurrentielle soit effective. Douze ans après la réforme, les résultats sont décevants. Alors que les écarts entre les primes restent très importants, la proportion d'assurés qui changent de caisse est faible chaque année: entre 4 et $5 \%$. Tout se passe comme si les assurés faisaient peu jouer la concurrence.

Comment interpréter cette inertie des consommateurs? Les faibles taux de mutations observés peuvent révéler des différences dans la qualité des services, d'importants coûts de mutation et/ou des pratiques de sélection des risques de la part des assureurs.

Dans cet article nous examinons le rôle potentiel des interactions entre le marché de l'assurance de base et celui des assurances supplémentaires. Certes, la loi interdit que les assurances de base et supplémentaires soient vendues comme des produits joints. En pratique cependant, certaines caractéristiques du marché de l'assurance supplémentaire peuvent avoir un impact sur le fonctionnement de la concurrence pour l'assurance de base. Notre enquête montre que $88 \%$ des individus ont souscrit au moins une assurance supplémentaire. Parmi eux, 91\% ont souscrit cette assurance auprès de la même compagnie que celle qu'ils ont choisie pour l'assurance de base. De facto, les deux marchés ne semblent pas véritablement séparés. En conséquence, il faut évaluer l'influence de la détention d'un contrat d'assurance supplémentaire sur les arbitrages du consommateur en matière d'assurance de base. Détenir une assurance supplémentaire peut exposer l'assuré à des pratiques de sélection des risques et augmenter ses coûts de mutation. Dans cette perspective, le rôle inhibiteur de l'assurance supplémentaire serait lié aux explications en termes de coûts de mutation et de sélection des risques mentionnées ci-dessus.

Nous reprenons la problématique d'un article précédent (Dormont et al. 2009) qui portait sur la période 1997-2000, laquelle se situe immédiatement après la réforme de la LAMal. Les faibles taux de mutations observés sur ce premier échantillon pouvaient être attribués aux délais nécessaires à la montée en régime du nouveau système de concurrence, au temps d'apprentissage des acteurs et à la mise en place des sites comparateurs de primes. Il était important d'examiner si l'ampleur des comportements de mutation reste réduite, et d'analyser si le rôle inhibiteur des assurances supplémentaires est confirmé sur une période plus récente, située plus de dix ans après la réforme. Notre objectif est aussi de présenter ces questions de structuration de l'assurance et le «modèle » de la concurrence régulée en assurance maladie à un public intéressé par la politique publique en France.

\section{Comment les assurances supplémentaires peuvent-elles inhiber les changements de caisse ?}

Trois caractéristiques du système suisse sont en jeu :

- il est possible de souscrire ses contrats supplémentaires chez l'assureur que l'on a choisi pour l'assurance de base ;

- souscrire l'assurance de base et ses assurances supplémentaires auprès de deux assureurs différents induit des coûts administratifs pour l'assuré à cause du double envoi des factures ;

- la loi qui régit les assurances supplémentaires permet une différenciation des contrats et autorise la sélection des assurés.

Ces règles entraînent que l'assurance supplémentaire peut influencer la mobilité des assurés dans l'assurance de base via quatre mécanismes.

(a) Le premier est un pur coût de mutation. Changer de caisse est coûteux en temps passé à collecter les informations et effectuer les démarches administratives. Comme la détention d'un contrat de base et d'un contrat supplémentaire auprès de deux assureurs différents induit des coûts administratifs additionnels, les assurés qui souhaitent changer de caisse pour l'assurance de base doivent aussi envisager de changer de caisse pour leur contrat d'assurance supplémentaire. Le coût de mutation est ainsi plus élevé pour un assuré qui dispose d'un contrat supplémentaire. Ce mécanisme est indépendant de l'état de santé de l'individu.

(b) La possibilité de sélection des risques sur le marché de l'assurance supplémentaire a pour conséquence qu'un assuré dont la santé n'est pas excellente s'identifie comme un mauvais 
risque et redoute d'être rejeté, s'il envisage de souscrire ses assurances supplémentaires auprès d'une nouvelle compagnie. Dans ce cas, la détention d'une assurance supplémentaire constitue un obstacle au changement de caisse dans l'assurance de base : la compagnie qui serait choisie pour l'assurance de base pourrait refuser de vendre un contrat d'assurance supplémentaire au nouvel arrivant ou demander en contrepartie le paiement d'une prime prohibitive. Ce mécanisme constitue un frein à la mutation lié à la détention d'une assurance supplémentaire qui ne doit s'observer que pour les individus qui jugent que leur santé est médiocre.

(c) Le troisième mécanisme suppose que les assureurs cherchent à retenir dans leur pool d'assurés les individus qui détiennent une assurance supplémentaire. En effet, la réglementation de l'assurance supplémentaire est peu contraignante : la possibilité de sélectionner les risques et l'absence de standardisation des contrats contribuent à réduire la pression concurrentielle. Dans cette perspective, les assureurs auraient intérêt à conserver les assurés munis d'une assurance supplémentaire, lesquels seraient particulièrement profitables quel que soit leur niveau de risque. Ce mécanisme implique que la possession d'un contrat supplémentaire joue négativement sur la probabilité de changer de caisse dans l'assurance de base indépendamment de l'état de santé subjectif des individus.

(d) Le quatrième mécanisme se réfère à l'idée de rétention des bons risques dans l'assurance de base. La détention d'une assurance supplémentaire peut être corrélée avec une meilleure santé et avec une faible consommation de soins de base en cas de maladie. Pourquoi les détenteurs d'assurance supplémentaire seraient-ils de bons risques ? Pensons aux médecines alternatives : des individus qui souscrivent ces contrats ${ }^{5}$ sont en principe plus réticents à la consommation des médicaments. Détenir une assurance supplémentaire peut aussi être associé à une plus grande attention portée à la santé et aux comportements de prévention. Si les compagnies délivrant l'assurance de base cherchent à retenir les bons risques, on devrait trouver que les détenteurs d'une assurance supplémentaire qui sont en bonne santé sont moins susceptibles de changer de caisse que les autres assurés ${ }^{6}$.

Comment les compagnies peuvent-elles sélectionner les risques alors qu'une telle pratique est interdite dans l'assurance de base ? Un exemple récent illustre les stratégies possibles. Une compagnie d'assurance active sur les marchés de l'assurance de base et de l'assurance supplémentaire a envoyé la lettre suivante à l'un de ses affiliés : «Pour vous remercier de votre fidélité, nous avons le plaisir de vous faire bénéficier des trois offres suivantes : 1 . une paire de lunettes pour un prix de 5 francs suisses (au lieu de 49,90 francs suisses) ; 2. un casque de ski pour un prix de 20 francs suisses (au lieu de 89,90 francs suisses) ; 3 . un contrat d'assurance privée d'hospitalisation en chambre double gratuit pendant la première année pour une période d'engagement minimale de 3 ans ». Ce courrier, daté du 12 septembre 2007, a été envoyé à un assuré qui n'avait pas eu de dépenses au titre de l'assurance de base au cours de l'année 2006. Des exemples de ce type sont régulièrement repris dans la presse helvétique.

\section{La concurrence a-t-elle entraîné une convergence des primes pour l'assurance de base?}

Contrats standardisés, primes intégralement à la charge des souscripteurs, diffusion publique de l'information sur les offres des différentes compagnies : la régulation introduite par la LAMal aurait dû faciliter les arbitrages des consommateurs et favoriser une réduction des écarts de primes pour l'assurance de base. Plus de dix ans après la réforme, force est de constater que les primes sont encore très disparates.

L'évolution de leur variabilité peut être examinée à partir de la base de données cantonales que nous avons constituée (cf. encadré 1). Notons $p_{j, c, t}$ la prime payée pour l'assurance de base offerte par la compagnie $j$ dans le canton $c$ en $t$. Pour l'année $t$, la variance totale des primes, $V\left(p_{j, c, t}\right)$, peut être décomposée en variances inter- et intra-cantons :

$V\left(p_{j, c, t}\right)=V\left(p_{., c, t}\right)-V\left(p_{j, c, t}-p_{., c, t}\right)$

La variance inter-cantons, $V\left(p_{. c, t}\right)$ résulte des différences de niveaux moyens des primes entre les cantons. Comme chaque citoyen doit s'assurer au sein de son canton, c'est à ce niveau géographique que joue la concurrence. Un impact

\footnotetext{
5. Dans notre enquête $45 \%$ des individus ont souscrit une supplémentaire couvrant les médecines alternatives.

6. En pratique la compagnie d'assurance identifie facilement ex post les bons et mauvais risques parmi ses affiliés, puisqu'elle observe leur utilisation des services de santé. Mais le point de vue de l'économètre, qui est le nôtre, est tout différent: nous n'observons qu'imparfaitement la consommation de soins et ne connaissons que la santé auto-évaluée. La détention d'une assurance supplémentaire croisée avec la bonne santé est ici utilisée comme un indicateur d'un bon risque.
} 
de la concurrence, s'il existe, doit se manifester par une réduction de la variance intra-cantons.

Nous avons examiné les valeurs annuelles des écarts-type des primes dans la dimension totale (les données annuelles des différents cantons sont empilées), inter- et intra-cantons, en utilisant la transformation logarithmique afin que la croissance des primes dans le temps n'affecte pas le diagnostic sur leur variabilité (cf. graphique I). Les statistiques sont pondérées afin de tenir compte de la taille des caisses en termes de nombre d'affiliés.

L'écart-type total du log des primes décroît très légèrement dans le temps, de 0,22 en 1996 à 0,18 en 2005. Mais ce mouvement résulte pour l'essentiel de la décroissance de l'écart-type inter-cantons ${ }^{7}$. Or, nous avons vu que la concurrence se joue à l'intérieur des cantons : un impact de la concurrence ne peut être observé qu'au seul niveau de l'écart-type intra-cantons. Celui-ci décroît très peu dans le temps, de 0,08 en 1996 à 0,07 en 2005. La convergence des primes depuis la mise en place de la LAMal est limitée.

L'évolution des premier et neuvième déciles permet de mieux comprendre de quoi est faite

7. On observe que plus de $80 \%$ de la variance du log des primes sont dus aux écarts inter-cantons, un résultat robuste à la prise en compte des différences de structure par âge et sexe des affiliés. Cette forte proportion de la variance inter-cantons reflète probablement des stratégies de fixation des prix par des compagnies qui sont généralement actives dans la plupart des cantons. Elle montre que restreindre la concurrence au périmètre du canton n'est pas sans inconvénient.

Encadré 1

\section{LES DONNÉES}

Notre base de données est construite à partir de deux sources : l'enquête Choix d'Assurance Maladie - IEMS-2007, menée auprès d'un échantillon représentatif des assurés en Suisse par l'Institut d'Économie et de Management de la Santé de l'Université de Lausanne; une base de données longitudinale construite au niveau cantonal par les auteurs. La réalisation de l'enquête a été conduite par l'Institut LINKS, sous la supervision de B. Dormont, P.-Y. Geoffard et $\mathrm{K}$. Lamiraud, lesquels avaient élaboré le questionnaire dans la continuité de celui de l'enquête OFAS (2001; cf. Dormont et al, 2009).

\section{L'enquête Choix d'Assurance Maladie IEMS-2007}

Cette enquête a été effectuée par téléphone entre juin et août 2007 auprès de 3016 assurés soumis à la LAMal et âgés de 26 ans ou plus. Une lettre de contact a été envoyée à un échantillon national aléatoire simple de ménages privés résidant en Suisse (tirage effectué par l'Office Fédéral des Statistiques). Une personne de 26 ans ou plus a ensuite été sélectionnée aléatoirement dans chacun des ménages éligibles (i.e. couverts par la LAMal) pour répondre au questionnaire.

L'enquête porte sur les choix d'assurance maladie. Les personnes interrogées doivent indiquer les choix effectués pour l'assurance maladie de base: le nom de la caisse, le montant de la franchise adoptée, l'option choisie (contrat avec choix restreint du médecin ou contrat permettant un choix non contraint du prestataire de soins), les raisons fondant le choix de la caisse, le montant de la prime. Pour les contrats d'assurance supplémentaires éventuellement souscrits, les individus doivent indiquer la nature des prestations supplémentaires couvertes, le nom de la caisse pour chacune des assurances supplémentaires contractées, ainsi que le montant de la prime.

Les assurés sont ensuite interrogés sur les changements qui ont pu intervenir entre 2003 et 2007 dans leur contrat d'assurance maladie de base (changement de caisse, de franchise ou d'options) et dans leurs éventuels contrats d'assurance supplémentaires (résiliation de certaines couvertures supplémentaires, signature de nouveaux contrats, changements de caisse). Le questionnaire demande aussi aux individus s'ils ont l'intention de changer de caisse dans un futur proche pour l'assurance de base.

Des informations sociodémographiques sur les personnes interrogées sont également collectées dans le cadre de cette enquête (âge, sexe, revenu, niveau d'éducation, nationalité, taille du ménage, activité professionnelle, code postal,) ainsi que des informations sur l'état de santé subjectif, quelques éléments sur l'utilisation des soins médicaux et le niveau des dépenses de santé.

\section{La base de données cantonale}

La base de données cantonale a été constituée à partir des registres de l'Office fédéral de la santé publique (OFSP) avec pour unité d'observation chacune des caisses d'assurance maladie opérant sur le marché de l'assurance de base sur la période 1996-2007. Ainsi, pour chaque caisse, dans chacun des cantons, nous disposons pour chaque année 1996 à 2007 du niveau de prime offert pour chaque type de contrat d'assurance de base (en fonction de la franchise et de l'option choisie) destiné aux adultes âgés de plus de 26 ans. Le nombre d'affiliés de chaque caisse dans chaque canton est également renseigné, mais tous contrats d'assurance de base confondus. 
la faible diminution de la disparité intra cantons. Nous avons calculé pour chaque canton le rapport du neuvième décile (D9) à la médiane (D5) de la distribution des primes (pondérées par le nombre d'affiliés par caisse), puis calculé la moyenne annuelle de ce rapport, avec une pondération tenant compte du nombre d'assurés dans chaque canton. Le même calcul est réalisé pour le rapport de la médiane au premier décile des primes (D5/D1). En début de période, des disparités en haut et en bas de la distribution des primes sont d'une ampleur similaire (cf. graphique II). On observe ensuite une réduction des disparités en haut de la distribution (D9/D5), suggérant qu'une pression s'exerce quand même à l'encontre des primes les plus élevées. Mais ce changement reste limité. Au total, la concurrence ne semble pas vraiment fonctionner.

\section{Peu d'individus changent de caisse d'assurance maladie}

Notre enquête révèle un taux de mutation assez faible: seulement $17,8 \%$ des individus de plus de 26 ans ont changé de caisse entre 2003 et 2007 , soit $4,5 \%$ par an (cf. tableau 1 ). Les intentions de changer de caisse sont aussi peu fréquentes : en $2007,11,6 \%$ des individus ont cette intention dans un futur proche.

Sur la période 1997-2000, 14,3\% des individus avaient changé de caisse, soit un taux annuel de 4,8\% (Dormont et al., 2009). Force est de constater que l'inertie des consommateurs est restée la même. Sur les années 1997-2000, situées juste après la réforme de 1996, il était possible d'imaginer qu'une période d'apprentissage était nécessaire pour que les consommateurs comprennent le fonctionnement du système et que les sites comparateurs de primes se mettent en place. Nos nouveaux résultats montrent que cette explication n'est pas tenable : un faible taux de mutation est observé de façon durable.

Une forte proportion d'individus - 87,6\% - a souscrit au moins une assurance supplémentaire. Une telle assurance correspond à différents

\section{Graphique I \\ Évolution de l'écart type total, inter-cantons et intra-cantons du log des primes pour l'assurance de base}

Écart-type

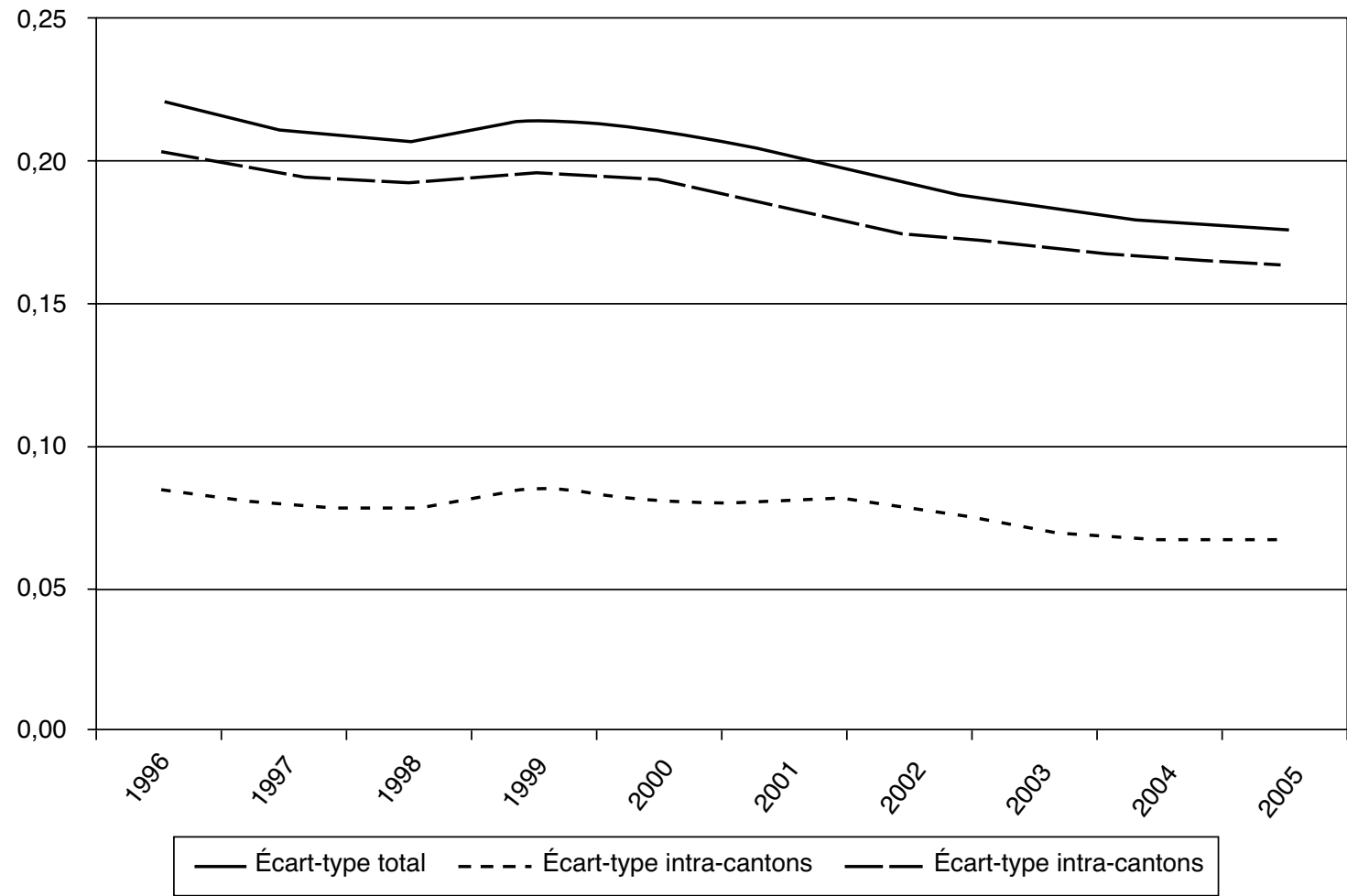

Champ : primes offertes par les assureurs maladie LAMAL pour le contrat d'assurance de base avec franchise basse, pondérées par la taille des caisses.

Source : base de données cantonale, calcul des auteurs. 
contrats possibles couvrant par exemple les soins dentaires, divers niveaux de confort à l'hôpital, les soins transfrontaliers, la médecine alternative, les arrêts maladie, etc. Un contrat très simple, appelé « Division commune Suisse entière ", permet d'étendre la couverture des soins hospitaliers aux soins délivrés dans tout le pays et non dans les hôpitaux du seul canton d'origine. Ce contrat peu coûteux est offert par la plupart des assureurs sans examen médical. En excluant les souscriptions à ce contrat, nous trouvons que $80,1 \%$ des individus ont souscrit une assurance supplémentaire.

Les primes sont indépendantes des caractéristiques de l'individu et de son revenu mais les ménages à faible revenu bénéficient d'une aide financière prenant la forme d'une subvention dont la générosité et les critères d'éligibilité sont définis au niveau cantonal. Dans l'échantillon, 16,8 \% des assurés bénéficient de cette subvention (cf. tableau 1). On observe par ailleurs que $37,2 \%$ des individus ont opté pour la franchise la plus basse (ce qui améliore la couverture, pour un niveau de prime majoré). Enfin, le montant de la subvention est calculé indépendamment de la caisse choisie. Par conséquent, il n'y a pas d'incitation ni d'obstacle au changement de caisse associé à la perception de cette subvention.

L'analyse descriptive des données de l'enquête montre aussi que la souscription d'un contrat d'assurance supplémentaire n'est pas liée au sexe de l'individu mais à son âge : comme les jeunes, les personnes de plus de 65 ans souscrivent une assurance supplémentaire dans une moindre proportion que les personnes d'âge intermédiaire (cf. tableau 2).

Détenir une assurance supplémentaire va de pair avec une moindre propension à changer de caisse. La probabilité de mutation est réduite d'un quart pour les individus qui ont une assurance supplémentaire (cf. tableau 3-A). L'intention de changer de caisse est aussi fortement réduite dans ce cas, presque divisée par deux.

\section{Graphique II \\ Évolution du rapport du neuvième décile à la médiane (D9/D5) et de la médiane au premier décile (D5/D1) de la distribution des primes pour l'assurance de base}

Rapport interdécile

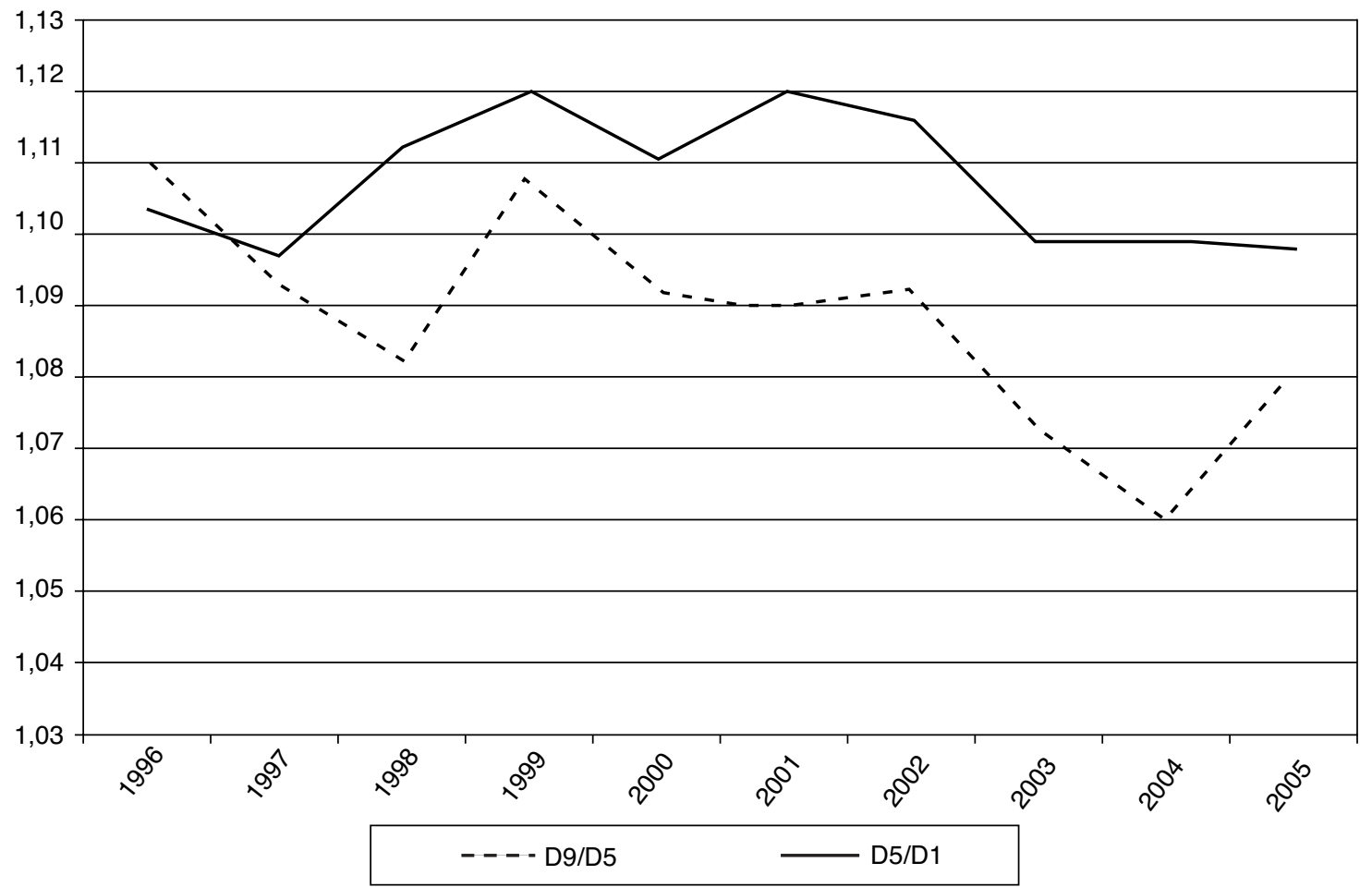

Champ : primes offertes par les assureurs maladie LAMAL pour le contrat d'assurance de base avec franchise basse, pondérées par la taille des caisses.

Source : base de données cantonale, calcul des auteurs. 
L'état de santé est mesuré par une note subjective $^{8}$ attribuée par l'individu lui-même à partir d'une grille en cinq catégories que nous avons réduite à trois catégories : état de santé mauvais, bon, très bon. $16,5 \%$ des individus de notre échantillon jugent leur santé mauvaise. À l'opposé, $38,4 \%$ des individus jugent leur santé très bonne (cf. tableau 1). Le niveau de revenu du ménage est fortement corrélé à l'état de santé subjectif : par rapport aux ménages à faible revenu, la probabilité de juger sa santé mauvaise est inférieure des deux-tiers pour un individu appartenant à un ménage de revenu intermédiaire. Pour les ménages les plus aisés, cette probabilité est réduite par un facteur supérieur à sept (cf. tableau 3-B). Détenir une assurance supplémentaire est aussi corrélé à un bon état de santé subjectif, sans que cette corrélation ne provienne de l'influence du revenu sur l'achat d'une assurance supplémentaire. En effet, les influences du revenu et de l'assurance supplémentaire sur la bonne santé subjective sont maintenues lorsque ces deux variables sont introduites simultanément dans l'estimation.

8. En économie de la santé, une littérature très abondante est consacrée aux relations existant entre état de santé subjectif et objectif, avec des discussions sur les difficultés à opérer même une distinction (par exemple, obtenir un diagnostic conduisant au constat objectif d'une maladie nécessite de la part de l'individu un jugement sur son état de santé le conduisant à aller consulter). Les études montrent que la note auto-attribuée est un excellent prédicteur des problèmes de santé.

Tableau 1

\section{Statistiques descriptives}

\begin{tabular}{|c|c|}
\hline \multicolumn{2}{|l|}{ Âge } \\
\hline$[27,35]$ & 12,7 \\
\hline$[36,50]$ & 35,0 \\
\hline$[51,65]$ & 29,3 \\
\hline$>65$ & 23,0 \\
\hline \multicolumn{2}{|l|}{ Revenu du ménage (CHF par mois) } \\
\hline$<5000$ & 34,4 \\
\hline $5000-8000$ & 30,8 \\
\hline$>8000$ & 34,8 \\
\hline Reçoit un subside (individuel) pour la prime (oui=1) & 16,8 \\
\hline Homme & 46,4 \\
\hline \multicolumn{2}{|l|}{ Niveau d'éducation } \\
\hline École obligatoire & 10,7 \\
\hline Maturité & 8,2 \\
\hline Apprentissage & 49,0 \\
\hline Formation professionnelle supérieure & 14,5 \\
\hline Université & 15,8 \\
\hline Milieu urbain & 69,1 \\
\hline \multicolumn{2}{|l|}{ État de santé subjectif } \\
\hline Mauvais & 16,6 \\
\hline Bon & 44,8 \\
\hline Très bon & 38,4 \\
\hline Franchise basse (300 CHF) pour l'assurance de base & 37,2 \\
\hline \multicolumn{2}{|l|}{ Contrat d'assurance supplémentaire } \\
\hline A au moins un contrat d'assurance supplémentaire & 87,6 \\
\hline A au moins un contrat d'assurance supplémentaire hormis « division commune Suisse entière » & 80,1 \\
\hline A changé de caisse d'assurance de base entre 2003 et 2007 & 17,8 \\
\hline A l'intention de changer de caisse dans un futur proche & 11,6 \\
\hline Nombre d'observations & 3016 \\
\hline
\end{tabular}

Champ : assurés en Suisse âgés de plus de 26 ans.

Source : enquête Choix d'Assurance Maladie IEMS-2007. 


\section{Modéliser les comportements de changement de caisse : spécification économétrique}

Pour étudier la mobilité des assurés, nous considérons une variable dichotomique $y_{i}$ telle que $y_{i}=1$ si l'individu $i$ a changé au moins une fois de caisse entre 2003 et 2007 et $y_{i}=0$ si l'individu $i$ est resté assuré auprès de la même caisse pour l'assurance de base au cours de cette période. Nous estimons le modèle :

$\left\{\begin{array}{l}y_{i}=1 \text { si } y_{i}^{*} \geq 0 \\ y_{i}^{*}=x_{i}^{\prime} \beta+s_{i} \gamma+u_{i}\end{array}\right.$

où $y_{i}^{*}$ représente le bénéfice net associé au changement de caisse. $x_{i}^{\prime}$ est un vecteur de caractéristiques individuelles et $s_{i}$ une variable dichotomique indiquant si l'individu détient $\left(s_{i}=1\right)$ ou non $\left(s_{i}=0\right)$ un contrat d'assurance supplémentaire. Le terme d'erreur $u_{i}$ est supposé suivre une loi normale.
Nous modélisons également la décision de souscrire au moins un contrat d'assurance supplémentaire? ${ }^{9}$. Le bénéfice net associé à la possession d'un tel contrat est représenté par la variable latente $s_{i}^{*}$ et la décision de souscrire un contrat d'assurance supplémentaire est définie par :

$\left\{\begin{array}{l}s_{i}=1 \text { si } s_{i}^{*} \geq 0 \\ s_{i}^{*}=z_{i}^{\prime} \alpha+v_{i}\end{array}\right.$

où $z_{j}^{\prime}$ est un vecteur de caractéristiques individuelles.

Peut-on penser que la souscription d'une assurance supplémentaire est une décision indépendante de celle de changer de caisse pour l'assurance de base ? Autrement dit, la variable $s_{i}$ indicatrice de la détention d'une assurance supplémentaire est-elle exogène dans l'équation (1) ? L'estimation séparée des modèles (1)

9. Hormis les contrats « Division commune Suisse entière ».

Tableau 2

Association entre la possession d'un contrat d'assurance supplémentaire et l'âge et le sexe de l'individu

\begin{tabular}{|c|c|c|c|}
\hline & $\begin{array}{l}\text { Sans contrat supplémentaire } \\
(\%)\end{array}$ & $\begin{array}{c}\text { Avec un contrat supplémentaire } \\
\text { (hormis " division commune Suisse entière ") } \\
(\%)\end{array}$ & $p$ \\
\hline \multicolumn{4}{|l|}{ Âge } \\
\hline$[27,35]$ & 22,72 & 76,50 & \\
\hline$[36,50]$ & 17,90 & 81,91 & \\
\hline$[51,65]$ & 14,71 & 85,07 & \\
\hline$>65$ & 26,98 & 73,02 & $<0,001^{*}$ \\
\hline Homme & 20,39 & 79,33 & \\
\hline Femme & 19,04 & 80,78 & $0,543^{\star \star}$ \\
\hline
\end{tabular}

Champ : assurés en Suisse âgés de plus de 26 ans.

Source : enquête Choix d'Assurance Maladie IEMS-2007.

Tableau 3-A

Association entre assurance supplémentaire et mutation ou intention de mutation

Régression logistique

\begin{tabular}{|l|c|c|c|}
\hline \multirow{2}{*}{} & \multicolumn{1}{|c|}{$\begin{array}{c}\text { Mutation } \\
\text { (au cours des 5 dernières années) }\end{array}$} & \multicolumn{2}{|c|}{$\begin{array}{c}\text { Intention de mutation } \\
\text { (dans un futur proche) }\end{array}$} \\
\cline { 2 - 4 } & $\begin{array}{c}\text { Odds } \\
\text { ratio }\end{array}$ & $p$ & $\begin{array}{c}\text { Odds } \\
\text { ratio }\end{array}$ \\
\hline $\begin{array}{l}\text { A au moins un contrat d'assurance supplémentaire } \\
\text { hormis « division commune Suisse entière » }\end{array}$ & 0,77 & 0,02 & 0,57 \\
\hline
\end{tabular}

Lecture : un individu détenant un contrat d'assurance supplémentaire a une probabilité de changement de caisse inférieure de $23 \%$ a ceux qui n'ont pas d'assurance supplémentaire.

Champ : assurés en Suisse âgés de plus de 26 ans.

Source : enquête Choix d'Assurance Maladie IEMS-2007, calcul des auteurs. 
et (2) peut conduire à des estimations biaisées si leurs termes d'erreur $u_{i}$ et $v_{i}$ sont corrélés. De façon générale, il est envisageable que ces termes contiennent tous deux des variables inobservables comme l'état de santé de l'individu ou son aversion pour le risque et soient donc corrélés. Notre spécification économétrique admet la possibilité d'une telle corrélation (cf. encadré 2), mais il faut avoir à l'esprit qu'elle peut ne pas être observée sur données suisses : en effet, la régulation sur les marchés de l'assurance de base qui impose une standardisation des contrats peut éliminer toute influence de l'état de santé ou de l'aversion pour le risque sur la décision de changer de caisse. Il est possible que ces variables inobservables n'affectent que le terme $v_{i}$.

\section{L'influence du revenu est de signe opposé pour l'assurance de base et l'assurance supplémentaire}

La probabilité de souscrire un contrat d'assurance supplémentaire n'est pas une fonction monotone de l'âge : son niveau le plus élevé est atteint pour les assurés âgés de 50 à 65 ans, et elle est moindre pour les moins de 35 ans et les plus de 65 ans. Les statistiques descriptives ont montré qu'un mauvais état de santé subjectif est corrélé à un revenu bas et à une faible propension à souscrire une assurance supplémentaire (cf. tableau 3-B). Dans l'estimation, l'information relative à l'état de santé subjectif est captée par la variable revenu : son influence sur la probabilité de souscrire un contrat d'assurance supplémentaire n'est plus significative lorsque le revenu et le niveau d'éducation sont introduits dans le modèle ${ }^{10}$.

Nous trouvons que le revenu joue positivement sur la probabilité de souscrire un contrat d'assurance supplémentaire. Ce résultat est en contradiction avec l'hypothèse "DARA » (decreasing absolute risk aversion) qui est la plus souvent retenue en théorie de l'assurance (Eeckhoudt, Gollier et Schlesinger, 2005) : stipulant que l'aversion pour le risque décroit avec le niveau de revenu, elle implique que l'assurance est un bien inférieur, autrement dit que la couverture demandée décroît lorsque le revenu augmente. En ce qui concerne l'assurance maladie, la couverture concerne le coût de services de santé, qui peuvent être nécessaires ex post. Ceci est vrai pour l'assurance de base comme pour l'assurance supplémentaire. Pour l'assurance supplémentaire cependant, les services de santé concernés peuvent être des biens supérieurs, voire des biens de luxe : par exemple, la disposition à payer pour une chambre individuelle à l'hôpital est vraisemblablement croissante avec le revenu. De ce fait, la demande de contrats d'assurance supplémentaire, qui couvrent ce type de services peut bien être, ex ante, une fonction croissante du revenu.

10. C'est pourquoi l'état de santé subjectif n'apparaît pas dans la partie inférieure du tableau 4.

Tableau 3-B

Association entre mauvais état de santé subjectif et plusieurs covariables

Trois régressions logistiques

\begin{tabular}{|c|c|c|c|c|c|c|}
\hline \multirow[t]{2}{*}{ Régression } & \multicolumn{2}{|c|}{ (i) } & \multicolumn{2}{|c|}{ (ii) } & \multicolumn{2}{|c|}{ (iii) } \\
\hline & Odds ratio & $p$ & Odds ratio & $p$ & Odds ratio & $p$ \\
\hline \multicolumn{7}{|l|}{ Revenu du ménage (CHF par mois) } \\
\hline$<5000$ & Ref. & Ref. & & & Ref. & Ref. \\
\hline $5000-8000$ & 0,35 & $<0,01$ & & & 0,36 & $<0,01$ \\
\hline$>8000$ & 0,14 & $<0,01$ & & & 0,14 & $<0,01$ \\
\hline \multicolumn{7}{|l|}{ Assurance supplémentaire } \\
\hline $\begin{array}{l}\text { A au moins un contrat d'assurance supplémentaire } \\
\text { hormis « division commune Suisse entière " }\end{array}$ & & & 0,635 & $<0,01$ & 0,762 & 0,02 \\
\hline
\end{tabular}

Lecture : la régression (i) examine la probabilité de juger sa santé mauvaise en fonction du niveau de revenu du ménage. Par rapport aux ménages aux revenus les plus faibles, un individu appartenant à un ménage de revenu intermédiaire a une probabilité de juger sa santé mauvaise inférieure de $65 \%$. Pour les individus appartenant aux ménages les plus aisés, la réduction est de $86 \%$.

La régression logistique (ii) montre qu'un individu détenant un contrat d'assurance supplémentaire a une probabilité de juger sa santé mauvaise inférieure de $36 \%$ à ceux qui n'ont pas d'assurance supplémentaire.

La régression (iii) est multivariée et examine l'impact du revenu et de la détention d'une assurance supplémentaire sur l'état de santé subjectif.

Champ : assurés en Suisse âgés de plus de 26 ans.

Source : enquête Choix d'Assurance Maladie IEMS-2007, calcul des auteurs. 
Pour l'assurance de base nous trouvons que le niveau de franchise choisi augmente avec le revenu $^{11}$. Ce résultat est conforme à l'hypothèse d'aversion au risque décroissante: les individus à haut revenu sont disposés à assumer une plus large part du risque financier associé aux soins de base. Au total, le revenu a des effets opposés sur la demande d'assurance de base et d'assurance supplémentaire. Notre interprétation n'est pas que l'aversion au risque varierait en fonction du type d'assurance concernée, mais que les fonctions de demande ex post pour les services couverts par l'assurance de base et l'assurance supplémentaire n'ont pas les mêmes effets revenu.

\section{La possible sélection des risques sur le marché de l'assurance supplémentaire contribue à l'inertie des assurés}

Quatre versions du modèle (1) sont estimées pour étudier les comportements de changement de caisse : on introduit tout d'abord seulement une variable indicatrice de la possession d'un contrat supplémentaire, en plus de l'âge, de la franchise et du milieu urbain (spécification I) ; ensuite on ajoute un indicateur de l'état de santé subjectif de l'assuré (spécification II). La spécification III retient une interaction entre ces deux variables, ce qui revient à admettre que l'impact de l'assurance supplémentaire sur la propension de l'individu à changer de caisse puisse varier en fonction de son jugement sur son état de santé. La spécification IV incorpore à la fois l'état de santé subjectif et son interaction avec l'indicatrice de détention d'une assurance supplémentaire.

On observe que la probabilité de changer de caisse diminue avec l'âge : par rapport au groupe de référence (36-50 ans), les assurés plus jeunes ont une probabilité plus élevée de changer de caisse. Du côté des plus âgés, les plus de 50 ans ont une moindre propension à changer de caisse et les plus de 65 ans sont encore plus inertes (cf. partie supérieure du tableau 4). Ce résultat est conforme à la littérature empirique sur la question (cf. encadré 3). Le lieu d'habitation

11. Ces résultats ne sont pas publiés ici car la demande d'assurance de base est en dehors du champ de cette étude, mais ils peuvent être communiqués à la demande.

Encadré 2

\section{ESTIMATIONS ÉCONOMÉTRIQUES}

Les expressions (1) et (2) forment un modèle récursif bivarié (Maddala, 1983). Si les termes d'erreur $u_{i}$ et $v_{i}$ ne sont pas indépendants, un estimateur convergent est obtenu en maximisant la vraisemblance définie à partir de la loi jointe des variables $s_{i}$ et $y_{i}$ (Probit bivarié). En revanche, si $u_{i}$ et $v_{i}$ ne sont pas corrélés, les deux équations peuvent être estimées de manière séparée.

Les conditions d'identification de ce modèle sont remplies. Plusieurs instruments déterminant la détention d'un contrat d'assurance supplémentaire n'expliquent pas le bénéfice à changer de caisse et sont donc exclus de l'équation (1) : le sexe, le revenu et le niveau d'éducation. Pour examiner si les instruments utilisés sont suffisamment corrélés à la variable indicatrice de la détention d'une assurance supplémentaire, nous avons estimé le modèle de probabilité linéaire correspondant. Nous trouvons une statistique de Fisher du test de la nullité des coefficients des instruments exclus égale à $F=12.29$, ce qui permet de conclure que les instruments utilisés ne sont pas faibles (Bound et al., 1995). Par ailleurs, le test de Sargan appliqué pareillement au modèle de probabilité linéaire correspondant permet de ne pas rejeter l'hypothèse nulle selon laquelle ces instruments étaient non corrélés avec $u_{i}(p=0.53)$. Le test de Sargan a été effectué sur la spécification III (cf. tableau 4).
Les modèles expliquant le changement de caisse et la souscription d'une assurance supplémentaire sont estimés séparément (cf. tableau 4) ou conjointement. Des constantes spécifiques aux cantons (effets fixes) sont incorporées aux équations afin de tenir compte de l'hétérogénéité inobservée entre les cantons. Les estimations réalisées sans effets fixes cantons sont disponibles sur demande. Elles ne donnent pas de résultats qualitativement différents. L'estimation du modèle bivarié conduit à une perte de précision pour l'impact de l'assurance supplémentaire. On obtient aussi une corrélation non significative entre les termes d'erreur $u_{i}$ et $v_{i}$ des deux équations, ce qui suggère qu'une estimation séparée des deux équations peut être légitime (les résultats de l'estimation jointe peuvent être communiqués au lecteur intéressé). Pour fonder une telle démarche, nous avons procédé à un test d'Hausman pour examiner si la variable $s_{i}$ (détention d'une supplémentaire) est une variable exogène dans le modèle expliquant le changement de caisse. Ce test, effectué sur le modèle de probabilité linéaire correspondant au modèle (1), permet de ne pas rejeter l'hypothèse d'exogénéité de $s_{i}$ (on a $p=0,89$ - le test a été effectué sur la spécification III (Tableau 4)). Nos commentaires se concentrent donc sur les résultats issus de l'estimation des équations séparées, en étudiant tout d'abord la décision de souscrire un contrat d'assurance supplémentaire (partie inférieure du tableau 4). 
(en milieu urbain ou rural) et le choix d'une franchise élevée ne sont pas significativement associés à la propension à changer de caisse.

La possession d'un contrat supplémentaire a un impact négatif sur la probabilité de changer de caisse, résultat inchangé lorsque nous contrôlons par l'état de santé subjectif (spécifications I et II). Lorsque l'impact de l'assurance supplémentaire peut varier en fonction de la santé subjective (spécification III), on trouve que la détention d'un contrat supplémentaire n'a pas d'effet significatif sur la probabilité de changer de caisse pour les individus jugeant leur santé bonne ou très bonne. En revanche, détenir une assurance supplémentaire a une influence

Tableau 4

Modèles Probit expliquant la probabilité de changer de caisse et la probabilité de souscrire à un contrat d'assurance supplémentaire

Variable expliquée : changement de caisse au cours des 5 années passées

\begin{tabular}{|c|c|c|c|c|c|c|c|c|}
\hline \multirow[t]{2}{*}{ Spécification } & \multicolumn{2}{|l|}{ (l) } & \multicolumn{2}{|l|}{ (II) } & \multicolumn{2}{|l|}{ (III) } & \multicolumn{2}{|l|}{ (IV) } \\
\hline & $\begin{array}{c}\text { Effet } \\
\text { marginal }\end{array}$ & $p$ & $\begin{array}{c}\text { Effet } \\
\text { marginal }\end{array}$ & $p$ & $\begin{array}{c}\text { Effet } \\
\text { marginal }\end{array}$ & $p$ & $\begin{array}{c}\text { Effet } \\
\text { marginal }\end{array}$ & $p$ \\
\hline \multicolumn{9}{|l|}{ Âge } \\
\hline$[27,35]$ & 0,100 & $<0,01$ & 0,100 & $<0,01$ & 0,101 & $<0,01$ & 0,100 & $<0,01$ \\
\hline$[36,50]$ & Ref. & Ref. & Ref. & Ref. & Ref. & Ref. & Ref. & Ref. \\
\hline$[51,65]$ & $-0,094$ & $<0,01$ & $-0,093$ & $<0,01$ & $-0,093$ & $<0,01$ & $-0,093$ & $<0,01$ \\
\hline$>65$ & $-0,155$ & $<0,01$ & $-0,151$ & $<0,01$ & $-0,152$ & $<0,01$ & $-0,151$ & $<0,01$ \\
\hline Milieu urbain & 0,028 & 0,086 & 0,027 & 0,101 & 0,027 & 0,10 & 0,027 & 0,10 \\
\hline Franchise $>300 \mathrm{CHF}$ & 0,016 & 0,288 & 0,010 & 0,485 & 0,011 & 0,47 & 0,011 & 0,49 \\
\hline $\begin{array}{l}\text { A au moins un contrat } \\
\text { d'assurance supplémentaire }\end{array}$ & $-0,036$ & 0,045 & $-0,038$ & 0,039 & - & - & & - \\
\hline \multicolumn{9}{|l|}{ État de santé subjectif } \\
\hline Mauvais & - & - & Ref. & Ref. & - & - & Ref. & Ref. \\
\hline Bon & - & - & $0,024^{a}$ & 0,272 & - & - & 0,009 & 0,82 \\
\hline Très bon & - & - & $0,035^{b}$ & 0,132 & - & - & 0,020 & 0,66 \\
\hline \multicolumn{9}{|c|}{ A un contrat d'assurance supplémentaire* et un état de santé subjectif } \\
\hline Mauvais & - & - & - & - & $-0,057^{c}$ & 0,02 & $-0,048$ & 0,22 \\
\hline Bon & - & - & - & - & $-0,034^{d}$ & 0,08 & $-0,032$ & 0,20 \\
\hline Très bon & - & - & - & - & $-0,024^{e}$ & 0,21 & $-0,032$ & 0,25 \\
\hline Nombre d'observations & 2869 & & 2862 & & 2862 & & 2862 & \\
\hline Log vraisemblance & $-1230,8828$ & & $-1227,1051$ & & $-1227,1146$ & & $-1227,0124$ & \\
\hline
\end{tabular}

Variable expliquée : a au moins un contrat d'assurance supplémentaire*

\begin{tabular}{|l|c|c|}
\hline \multicolumn{1}{|c|}{ Spécification } & \multicolumn{2}{c|}{ (i)' } \\
\hline \multicolumn{1}{|c|}{} & $\begin{array}{c}\text { Effet } \\
\text { marginal }\end{array}$ & $p$ \\
\hline Âge & $-0,061$ & 0,01 \\
{$[27,35]$} & $R e f$. & Ref. \\
{$[36,50]$} & 0,039 & 0,03 \\
{$[51,65]$} & $-0,013$ & 0,53 \\
$>65$ & & \\
\hline Revenu du ménage (CHF par mois) & Ref. & Ref. \\
$<5000$ & 0,073 & $<0,01$ \\
$5000-8000$ & 0,131 & $<0,01$ \\
$>8000$ & $-0,041$ & $<0,01$ \\
\hline Homme & & \\
\hline
\end{tabular}


négative sur la propension à changer de caisse pour les individus jugeant leur santé mauvaise.

Ces résultats suggèrent que ce sont les croyances des assurés quant aux pratiques de sélection des risques sur le marché de l'assurance supplémentaire qui expliqueraient une moindre mobilité dans l'assurance de base pour les détenteurs d'un contrat d'assurance supplémentaire: le mécanisme (b) décrit plus haut serait à l'œuvre. Soulignons que l'effet significatif des variables croisées ne capture pas un effet direct de l'état de santé subjectif sur la propension à changer de caisse. Les résultats de la spécification II montrent en effet que l'état de santé subjectif n'a pas d'impact direct sur la probabilité de changer de caisse ${ }^{12}$.

Les estimations suggèrent aussi que les mécanismes (c) et (d) envisagés plus haut ne jouent pas de rôle significatif dans l'explication de l'inertie des consommateurs. Si le mécanisme (c) était à l'œuvre, l'impact de l'assurance supplémentaire sur la probabilité de changer de caisse ne dépendrait pas de l'état de santé des individus. Le mécanisme (d) implique qu'un très bon état de santé devrait réduire la probabilité de changer de caisse et/ou accentuer l'impact négatif de la possession d'un contrat supplémentaire sur le changement de caisse. Or, les résultats vont à l'encontre de ces prédictions : introduit isolément, l'état de santé subjectif n'a pas d'effet significatif sur la probabilité de changer de caisse et la détention d'un contrat supplémentaire n'a pas d'impact significatif sur la probabilité de changer de caisse pour les individus en très bon état de santé. Le fait que ces individus, qui n'ont pas de sélection à craindre, n'ont pas leur propension à changer de caisse freinée par la détention d'une complémentaire réfute enfin l'idée du double coût de mutation et donc le mécanisme (a).

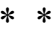

De nombreux pays ont cherché à introduire des mécanismes concurrentiels en assurance maladie, tout en instaurant une régulation permettant d'éviter la sélection des risques, de garantir une solidarité entre malades et bien portants et de faciliter l'accès à l'assurance pour les ménages à bas revenu. Le modèle de la « concurrence régulée » est ainsi appliqué pour l'assurance maladie de base en Suisse et dans de nombreux pays. Ce modèle inspire la réforme Obama aux États-Unis. Il pourrait être appliqué en France pour les assurances complémentaires, dans l'hypothèse où leur contribution à la couverture des soins continuerait à croître.

12. Voir en bas du tableau 4 les tests examinant la significativité jointe des coefficients des variables «bonne santé » et " très bonne santé » pour la spécification II, ainsi que le test du rapport de vraisemblance comparant les spécifications III et IV.

Tableau 4 (suite)

\begin{tabular}{|l|c|c|}
\hline Niveau d'éducation & \multicolumn{1}{|c|}{ Ref. } & \multicolumn{1}{|c|}{ Ref. } \\
École obligatoire & 0,103 & $<0,01$ \\
Maturité & 0,051 & 0,02 \\
Apprentissage & 0,064 & 0,02 \\
Formation professionnelle supérieure & 0,057 & 0,04 \\
Université & 0,014 & 0,37 \\
\hline Franchise > 300 CHF & 2885 & \\
\hline Nombre d'observations & $-1313,697$ & \\
\hline Log vraisemblance & & \\
\hline
\end{tabular}

* hormis " division commune Suisse entière "

$\mathrm{ab}$ Test de Wald $\mathrm{HO}$ : les coefficients des variables « bon » et « très bon » état de santé $=0: \mathrm{p}=0,32$

cde Test de Wald de H'0 : les coefficients des trois variables croisées sont égaux : $p=0,05$

cd Test de Wald de H" 0 : les coefficients des deux variables croisées sont égaux ; $p=0,04$ Toutes les régressions incorporent des effets fixes canton.

Lecture : un test de rapport de vraisemblance montre que le modèle IV n'apporte pas d'amélioration significative par rapport au modèle III ( $\mathrm{p}=0.903$ ). Dans le modèle IV, l'ajout des variables de santé rend non significatifs les coefficients des variables de santé croisées avec la détention d'une supplémentaire. Cependant, cette non significativité est due à la perte de précision causée par l'introduction des variables de santé : les coefficients estimés des variables croisées ne changent pas beaucoup entre les modèles III et IV mais leurs écarts-type estimés augmentent de $33 \%$ à $60 \%$. C'est pourquoi nous nous centrons sur les résultats du modèle III.

Champ : assurés en Suisse âgés de plus de 26 ans.

Source : enquête Choix d'Assurance Maladie IEMS-2007, calcul des auteurs. 
En effet, les assurances complémentaires en accès individuel sont peu régulées en France. L'achat d'une assurance est facultatif et passe par le paiement d'une prime généralement indépendante du revenu et souvent croissante avec l'âge. Dans ce cas, le financement de l'assurance est dégressif: il représente 2,9\% du revenu des ménages les plus aisés (décile supérieur des revenus) mais $8 \%$ du revenu des ménages les plus modestes (décile inférieur audessus du plafond CMU). En outre, la concurrence entre les organismes, jointe au caractère facultatif de l'assurance complémentaire, incite les assureurs à segmenter les contrats et à ajuster les primes au plus près des dépenses prévues pour les individus. Au total, les primes peuvent

Encadré 3

\section{LA MOBILITÉ DES ASSURÉS DANS LES SYSTÈMES DE CONCURRENCE EN ASSURANCE MALADIE}

Certains pays occidentaux ont confié la gestion du risque maladie de base à des sociétés privées exerçant leur activité dans un environnement concurrentiel : la Suisse, les Pays-Bas, l'Allemagne, la Belgique, Israël et les États-Unis. La concurrence entre assureurs doit en théorie conduire à une meilleure efficacité productive (meilleure qualité au moindre coût). En pratique, un système concurrentiel ne fonctionne que si les consommateurs "font jouer la concurrence" en choisissant les assureurs les plus efficaces. Or, de nombreux articles étudiant la mobilité des assurés dans les systèmes de concurrence en assurance santé soulignent la relative inertie des consommateurs et essaient d'expliquer leur faible mobilité.

Quel que soit le pays considéré, les assurés changent peu d'assureurs. En Suisse, moins de $5 \%$ des assurés changent d'assureur chaque année (Dormont et al., 2009). Les Pays-Bas ont introduit en 2006 un système de compétition encadrée qui présente de nombreuses similitudes avec le système suisse. La mobilité des assurés a été élevée au cours de l'année 2006 (29\%) mais les taux de changement de caisse sont redevenus faibles au cours des années suivantes (Lako et al., 2011). Aux États-Unis, les entreprises offrent à leurs employés le choix entre plusieurs plans d'assurance mais les taux de mobilité des assurés ne dépassent pas $5 \%$ par an (Samuelson et Zeckhauser, 1988).

De nombreux articles montrent que la propension à changer de caisse est plus forte pour les assurés jeunes, en bonne santé et/ou avec un niveau d'éducation élevé (Strombom et al., 2002). La mobilité des assurés est également influencée par les caractéristiques des assureurs et en particulier par les niveaux des primes. De nombreux travaux estiment l'élasticité prix de la demande. Cette dernière varie de - 8.4 à - 0.1 selon les études (Schut et al., 2003 ; Laske-Aldershof et Schut, 2003 ; Buchmueller, 2000 ; Buchmueller et Feldstein, 1997 ; Royalty et Solomon, 1999). En Suisse, à partir de données de marché, Beck et al. (2003) estiment que l'élasticité-prix est comprise entre -2.1 et - 1.0. Plus récemment, Tamm et al. (2007) ont montré que les élasticités-prix sont faibles à court terme et élevées à long terme. Aux États-Unis où les caisses peuvent contracter avec les fournisseurs de soins de leur choix (managed care), la littérature étudie aussi l'impact de la qualité des soins sur la mobilité des assurés. Beaulieu (2002) montre que l'information sur la qualité des soins influence la mobilité des assurés mais ce résultat n'est pas retrouvé par Abraham (2006).

Pourquoi la mobilité des assurés est-elle si faible au total ? Différentes raisons ont été étudiées dans la littérature :

- Les coûts de changement liés par exemple aux contraintes administratives de la démarche ou aux différences de qualité de service entre les caisses. En Allemagne, Nuscheler et Knaus (2005) soulignent l'importance des coûts de changement, qui sont plus élevés pour les individus en mauvaise santé. Aux États-Unis, un assuré peut être réticent à changer de caisse parce que ce changement implique aussi un changement de médecin (Abraham, 2006).

- Le manque d'information ou la difficulté d'accès à I'information (McCarthy et Tchernis, 2010).

- L'excès de choix qui fait référence à l'incapacité des consommateurs à prendre des décisions dans des situations de choix multiples et complexes (Eppler et Mengis, 2003 ; Samuelson et Zeckhauser, 1988). Ce phénomène semble freiner la mobilité des assurés suisses dans l'assurance maladie de base. Frank et Lamiraud (2009) montrent que lorsque le nombre d'assureurs augmente, la probabilité qu'un assuré change de caisse diminue et ce, indépendamment du taux de concentration des caisses.

- Le biais de statu quo qui est associé à la tendance à surestimer les désavantages et à sous-estimer les gains associés à un changement de situation (Tversky et Kahneman, 1991). C'est ainsi que les assurés persistent dans leur choix initial (Samuelson and Zeckhauser, 1988; Strombom, Buchmueller et Feldstein, 2002 ; Frank et Lamiraud, 2009).

- Le rôle de l'assurance supplémentaire. Les travaux sur données suisses (Dormont et al., 2009) et néerlandaises (Roos et Schut, 2012) montrent que la possession d'un contrat d'assurance supplémentaire réduit la mobilité dans l'assurance de base pour les individus en mauvaise santé.

- Les pratiques de sélection des risques dans l'assurance de base (attirer les bons risques ou dissuader les mauvais risques et les inciter à changer de caisse). De telles pratiques, si elles existent, rendent inopérant le mécanisme de concurrence par les prix et constituent un frein important à la mobilité des assurés. Nuscheler et Knaus (2005) ne retrouvent pas l'existence de ce phénomène sur données allemandes. 
atteindre des niveaux prohibitifs pour certains individus et les contrats proposés peuvent être difficilement compréhensibles en termes de couverture offerte. Un renoncement à l'assurance complémentaire est possible, pouvant entraîner un problème d'accès aux soins.

Tout suggère qu'en France la concurrence entre les organismes d'assurance complémentaire ne joue pas en faveur de l'offre d'une bonne couverture à moindre coût, mais conduit pour l'essentiel à une segmentation des contrats pour s'attirer des publics particuliers. Cette différenciation accrue rend l'offre peu lisible pour les consommateurs, lesquels peuvent difficilement faire jouer la concurrence. La question de la définition d'un contrat standard pourrait donc se poser pour les assurances complémentaires en France. Or la définition d'un contrat standard pourrait conduire à l'émergence d'une offre d'assurance «sur-complémentaire» pour les biens non inclus dans ce contrat, avec les effets potentiellement perturbateurs que l'on observe en Suisse.

L'expérience de la Suisse permet d'étudier les propriétés du modèle de concurrence régulée. Notre analyse montre que la faible mobilité des assurés résulte de la coexistence de deux marchés d'assurance maladie soumis à différentes règles de fonctionnement: le marché de l'assurance de base, où la sélection des risques est interdite, et celui de l'assurance supplémentaire, où elle est autorisée. Or, bien que séparés en droit, ces deux marchés sont intimement liés en pratique. Nous trouvons que la propension à changer de caisse est beaucoup plus faible chez les détenteurs d'une assurance supplémentaire. Or, cet effet négatif de l'assurance supplémentaire n'est observé que chez les individus qui estiment que leur santé n'est pas excellente. Le mécanisme serait le suivant : étant donné qu'il est préférable pour des raisons pratiques d'avoir son assurance de base et son assurance supplémentaire dans la même caisse, détenir une assurance supplémentaire crée un obstacle à la mobilité sur l'assurance de base pour l'individu qui considère que sa santé n'est pas suffisamment bonne pour être certain de pouvoir souscrire une assurance supplémentaire dans la nouvelle caisse. On trouverait là l'explication de la relative inertie des consommateurs. Le lien de fait existant entre assurance de base et assurance supplémentaire rend inopérants les mécanismes mis en place pour favoriser la concurrence. Le droit de sélectionner les candidats à la souscription pour l'assurance supplémentaire nuit à la concurrence sur l'assurance de base.

La régulation de la concurrence en assurance maladie trouve ainsi sa limite dans la liberté plus grande accordée sur le marché de l'assurance supplémentaire, une leçon pour tout législateur qui voudrait mettre en place une concurrence régulée en assurance maladie.

\section{BIBLIOGRAPHIE}

\begin{abstract}
Abraham J.M., Feldman R., Carlin C. et Christianson J. (2006), " The effect of quality information on consumer health plan switching: Evidence from the Buyers Health Care Action Group », Journal of Health Economics, vol. 25, pp. 762-781.
\end{abstract}

Beaulieu N.D. (2002), " Quality information and consumer health plan choices », Journal of Health Economics, vol. 21, pp. 43-63.

Beck K., Käser-Meier U. et Gelpke V. (2003), «Analyzing consumer mobility in Swiss health insurance ", Paper presented at the $4^{\text {th }}$ iHEA World Congress on Health Economics, San Fransisco, June 15-18, Mimeo CSS Insurance.

Bound J., Jaeger D.A. et Baker R. (1995), « Problems with Instrumental Variables Estimation
When the Correlation Between the Instruments and the Endogenous Explanatory Variables is Weak », Journal of the American Statistical Association, vol. $90, \mathrm{n}^{\circ} 430$, pp. $443-450$.

Buchmueller T.C. et Feldstein P.J. (1997), «The Effect of Price on Switching Among Health Plans", Journal of Health Economics, vol. 16, pp. 231-247.

Buchmueller T.C. (2000), «The Health Plan Choices of Retirees under Managed Competition », Health Services Research, vol. 35, $\mathrm{n}^{\circ}$ 5, pp. 949-976.

Colombo F. et Tapay N. (2004), « Private Health Insurance in OECD Countries: The Benefits and Costs for Individuals and Health Systems ", OECD Health Working Paper $n^{\circ} 15$. 
Dormont B., Geoffard P.Y. et Lamiraud K. (2009), «The influence of supplementary health insurance on switching behaviour: evidence from Swiss data », Health Economics, vol. 18, $\mathrm{n}^{\circ} 11$, pp. 1339-1356.

Eppler M.J. et Mengis J. (2003), «A Framework for Information Overload Research in Organizations. Insights from Organization Science, Accounting, Marketing, MIS, and Related Disciplines », Paper $n^{\circ} 1$ Università della Svizzera italiana.

Frank R.G. et Lamiraud K. (2009), « Choice, Price competition and Complexity in Markets for Health Insurance », Journal of Economic Behavior and Organization, vol. 71, $\mathrm{n}^{\circ} 2$, pp. 550-562.

Lako C. J., Rosenau P. et Daw C. (2011), « Switching Health Insurance Plans: Results from a Health Survey "), Health Care Analysis, vol. 19, $\mathrm{n}^{\circ} 4$, pp. 312-328

Laske-Aldershof T. et Schut F.T. (2003), «Switching behaviour of consumers in Dutch social health insurance », Paper presented at the $4^{\text {th }}$ iHEA World Congress on Health Economics, San Francisco, June 15-18.

McCarthy I.M. et Tchernis R. (2010), « Search Costs and Medicare Plan Choice », Health Economics, vol. 19, pp. 1142-1165.

Nuscheler R. et Knaus T. (2005), « Risk Selection in the German Public Health Insurance System », Health Economics, vol. 14, n 12 , pp. 1253-1271.

OFS (2009), « Coût et Financement du système de santé en 2007 », Office Fédéral de la Statistique (OFS).
Roos A.F. et Schut F.T. (2012), «Spillover effects of supplementary on basic health insurance: evidence from the Netherlands ", European Journal of Health Economics, vol. 13, $\mathrm{n}^{\circ} 1$, pp. 51-62.

Royalty A.B. et Solomon N. (1999), « Health Plan Choice: Price Elasticities in a Managed Competition Setting ", The Journal of Human Resources, vol. 34, $\mathrm{n}^{\circ} 1$, pp. 1-41.

Samuelson W. et Zeckhauser R. (1988), « Status Quo Bias in Decision Making », Journal of Risk and Uncertainty, vol. 1, $\mathrm{n}^{\circ} 1$, pp. 7-59.

Schut F.T., Greb S. et Wasem J. (2003), «Consumer Price Sensitivity and Social Health Insurer in Germany and the Netherlands », International Journal of Health Care Finance and Economics, vol. 3, pp. 117-138.

Staiger D. et Stock J.H. (1997), « Instrumental variables regression with weak instruments», Econometrica, vol. 65, pp. 557-586.

Strombom B.A., Buchmueller T.C. et Feldstein P.J. (2002), « Switching costs, price sensitivity and health plan choice », Journal of Health Economics, vol. 21, pp. 89-116.

Tamm M., Tauchmann H., Wasem J. et Greß S. (2007), «Elasticities of market shares and social health insurance choice in Germany: a dynamic panel data approach », Health Economics, vol. 16, pp. 243-256.

Tversky A. et Kahneman D. (1991), «Loss Aversion in Riskless Choice: A Reference Dependent Model», Quarterly Journal of Economics, vol. 106, pp. 1039-1061. 
